\title{
CONFLICT BETWEEN INDIVIDUALS AND GROUPS IN A CHANGING ORGANIZATION - A CONCEPTUAL REVIEW
}

\author{
Fuad Cholisi dan Sri Mulyono
}

\begin{abstract}
The traditional view sees conflict as something negative and destructive, and therefore should be avoided. Contradictorily, the human relations view holds that conflict is a natural and inevitable part of organizational process and operation, which is not necessarily a negative thing. If conflict is handled in a constructive manner, it can lead to positive outcomes. This essay aims to explore how an organizational change can result in conflict between individuals or groups, the nature of the arising conflict, and some proposed formulations for conflict resolution. Organizations apparently need to keep changing because they have to continue to adapt to the continually changing situation and environment. Whilst research works generally reveals that conflict resulting from an organizational change is unavoidable due to different individual interpretations of facts and differences in expectations, the source of organizational changes may include power and politics, organizational structures, cultural differences, and environmental change. No matter which view of conflict one holds, it is widely agreed that conflict needs to be resolved in order to improve the performance of the organization involved, and among the proposed strategies of managing conflicts are the nine formulations proposed by Mullins and the Thomas's Model of Conflict-Handling Styles.
\end{abstract}

Key words: conflict, traditional view, human relations view, organizational change, power, organizational structure, culture, environment, resolution model.

In a world of relentless change, organizations are continually confronted with situations of uncertainty, and strategy for change or managing change has inescapably been a household issue for organizations to deal with if they are to survive. Unfortunately, organizations are not machines, but they are comprised of people of different backgrounds, interests, and behaviors. Organizational change efforts frequently run into conflict resulting from either individual or group differences.

Over the years conflict has been of particular interest to managers, management strategists, and behavioral scientists for studies and discussions due to its important role in ensuring the success of organizational changes and performance. When coming to the meaning of conflict, however, there exists a main dividing line between two different views: (1) conflict is seen as a danger with its destructive capability, and conflict as a phenomenon which can have constructive or 
destructive effects depending on how it is managed (Thomas, 1976; Schelling, 1980; Mullins, 1999); (2) later approaches to conflict generally take the view that conflict is inevitable, and is a natural occurrence in all organizations. Two crucial questions can arise at this point. First, as the title of this essay implies, is it true that in the times of change in an organization, conflict between individuals and groups is inevitable? Secondly, how should organizations manage a conflict in order to minimize its destructive effects, and benefit by its constructive effects?

This essay will address these questions by first looking at the reasons why organizations need to change, before discussing the first main part of the essay: the meanings, the nature and the sources of conflict. By then the essay will hopefully have answered the first question while the remaining main part of the essay will be focused on answering the second question.

\section{Why Organizations Need To Change}

The reasons why organizations need to change are mainly based on the open system concepts to study individuals and groups in organizational psychology (Alderfer, 1976). Activities within an organization as an open system are shaped largely by external factors, the environment in which it is operating. Through exchange and contacts with the external environment, an organization can anticipate its ends, guarantee its life, or even increase its dynamism. In fact, environmental changes are the main external triggers for organizational change. Population, ecology, state regulations or government intervention, culture, labor markets, technology, and resources are among the most prominent factors. Senior (1997) has mentioned that such environmental factors are becoming more uncertain, together with the quickening of the pace of change and the unpredictability of the future. It is in this kind of environment that organizations have to operate. Consequently, they have to continue to adapt to the continually changing environment, and to go on changing perpetually. As argued by Handy (1993), whether it is for individuals or organizations, change is a necessary condition for survival.

In addition to such triggers for change coming from external environment, many writers and researchers have suggested that organizational change may also be 
triggered by the forces within the organization itself. This is what they call internal triggers for change (Senior, 1997). With this type of triggers in mind, organizations have consequently to contend with both external and internal triggers for change.

Regarding the environment where organizations operate, Senior (1997) has argued that there are actually three types of environments that together make up the total operating environment, namely temporal environment, external environment, and internal environment. She has developed a model whereby she describes an organization as a system that operates in multi-dimensional environments, i.e., those three types of environments. At the core of the system is the organization itself, which consists of the formal sub-system and the informal sub-system. In Senior's account, all of the three types of environments have in some way their respective impacts on the organization in that they force it to accomplish changes if it is to survive. As confirmed by Mullins (1999), "change is an inescapable part of both social and organizational life." It may boost an organization's performance through stronger relationships and better understanding of others if it is handled in a constructive way.

\section{Conflict in the Time of Change}

As with the case of many other scientific terms, it is not easy to define what conflict is. Robbins (1994) defines conflict as "a process in which an effort is purposely made by A to offset the efforts of B by some form of blocking that will result in frustrating B in attaining his or her goals or furthering his or her interests". Similar to this definition is the one given by Thomas (1976), where conflict is defined as "the process which begins when one party perceives that the other is frustrated, or is about to frustrate, some concern of his". In a rather straightforward way, Mullins (1999) defines conflict as "behavior intended to obstruct the achievement of some other person's goals." These definitions convey three important points. First, it takes the form of a process. Second, it involves two parties who are in disagreement or opposition, and finally, it results in a kind of frustration or obstructed achievement of some goals. 
According to Senior (1977), conflict comes in different guises according to its degree of seriousness and capacity to disrupt or, in some cases, improve a difficult situation. Conflict may arise due to different individual interpretations of facts and differences in expectations. In other words, conflict arises as a natural consequence of different opinions and ideas of different people. This can particularly happen when the change involves an organizational structure, which may threaten personnel position or end up in redundancy. An example of conflict situation is where two managers are in contest with each other to avoid compulsory redundancy. This is an example of individual conflict, while an example of conflict between groups could be a situation where two different departments in a company such as marketing department and production department are trying to protect their respective interests without taking care of the negative impact to the company. This may be due to lack of organizational bonding or sense of belonging because of weak organizational culture. Many cases have also shown that when a change occurs in an organization, the organization's unity and interpersonal as well as inter-group relationships are threatened.

\section{Contrasting Views of Conflict}

It is interesting to find that there has been conflict over how conflict is viewed by different approaches. The traditional view assumes that conflict is destructive, and therefore should be avoided. In this view, conflict is generally associated with negative features and situations that will contribute to inefficiency, ineffectiveness, counter-productivity, and other negative consequences (Robbins, 1994; Mullins, 1999). In this approach, conflict is considered as dysfunctional in that it will hinder the organization's performance.

In contrast, the human relations approach takes the view that conflict is not necessarily a bad thing and is inevitable in any organization, particularly in the times of change. Therefore, this approach has advocated acceptance of conflict, and believes that there are even times when conflict can have potentially positive outcomes. As Mullins (1999) argues, conflict can be seen as a 'constructive' force and in certain circumstances it can be welcomed or even encouraged. Rather than 
being seen as dysfunctional, conflict is viewed from a different angle and considered as functional, which may improve organizational performance. For example, it can be seen as an aid to incremental improvement in organization design and functioning, and to the decision making process. Conflict can also be an agent for evolution, and for internal as well as external change.

What Mullin has stated above is in support of what is currently popular and known as the interactionist view (Robbins, 1994). This view has gone one step further from the human relations view in that it encourages conflict rather than just accepting it, and therefore, it has a major contribution in encouraging group leaders to maintain an ongoing minimal level of conflicts.

The last four decades have seen a more general recognition that interpersonal and inter-group conflicts often serve a useful function. As Thomas (1976) has suggested, a moderate degree of conflict should be viewed as a positive phenomenon required by organizations to maintain their optimal level of stimulation rather than as costs. Referring to the current trend in theories of motivation, he has supported his argument by confirming that people may welcome or seek out the novelty of divergent opinions, the challenge of competition, and sometimes the excitement of overt hostilities.

\section{Sources of Conflict in the Times of Change}

Various lists of sources of conflict have been produced by different writers (Thomas, 1976; Handy, 1993; Robbins, 1994; Senior, 1997; Mullins, 1999). In this essay, however, only those which are relevant to the topic of this essay will be discussed as presented in the following.

\section{Power and Politics}

Mullins (1999) has argued that in addition to being systems of hierarchical structure, work organizations are also systems of social relationship, status and power. Power can be interpreted either in terms of control of influence over the behavior of other people with or without their consent, or in terms of the extent of the influence which can actually be exercised over other people, and may include influence over objects as well as 
people, whilst politics, according Senior (1997), is the use of power which is not part of someone's formal organizational role. She has explained that as the organizational world is made up of human beings with their own set of values, interests and beliefs, what happens in organizations is frequently more characterized by such individual interests and beliefs.

The argument above is supported by Handy (1993), who observed the tendency for individuals and groups to pursue courses of actions which promote their interests, regardless of the organization's formal goals and objectives. This phenomenon of individuals and groups pursuing differing interests and battling each other throughout an organization has resulted competition for power within the organization, which in turn may lead to conflict. In short, conflict can arise from excessive efforts of using power to control others for individual or group interests.

\section{Organizational Structures}

Organization structure may become a source of conflict when there is power imbalance in hierarchical structures. In the newly adopted matrix system, for example, a manager may be confused by the dual chain of command, which means that he should report to both his vertical line senior manager and to a divisional head horizontally. This can be a potential source of conflict. As Johnson and Scholes (1989) argued, matrix structure has a real problem of obscurity of who is responsible for what.

Regarding the conflict triggered by a change in organizational structure, the experience of Zeton Ltd., an engineering company based in Kuala Lumpur, is a good example (Mullins, 1999). Tommy Lee, the Chief Executive of the company, faced a dilemma when he had to restructure the organization of his company to meet the demand for a possible export market as a supplier to a German car manufacturer. There was certainly a conflict between Lee's personal interest in keeping his strong control over the company under the old, bureaucratic system and the need for changing the organization structure to a leaner and more efficient organization in order to cope with the expanding market demands and the growth of the company. 


\section{Cultural Differences}

Cultural differences can be a potential source of conflict when people from different nationalities and societies work together. As Senior (1997) has pointed out, "conflict can arise through misunderstandings or through inappropriate behavior on the part of those with one set of cultural characteristics towards those with other characteristics." A kind of cultural shock may be experienced, for example, by an expatriate who has just been assigned to be a manager in a foreign country away from his own country. Similarly, a change in organizational culture, for networking reasons for example, can trigger a conflict between individuals as well as groups within the organization because of resistance to change found in one or more of the parties involved.

Robbins (1994) argues that national culture would to some degree influence one's approach to handling conflict. Referring to Hofstede's dimensions of national culture, he mentioned the Americans as an example for the characteristics of a society marked by relatively low uncertainty avoidance and high masculinity rankings. The implication of these characteristics for organization is that their organizations tend to be more open and flexible, because the cultural climate of low uncertainty avoidance and high masculinity tends to shape a society that is open, direct, and competitive. When confronted to an organizational culture that is marked by high power distance and high uncertainty avoidance, for example, these people will presumably experience cultural conflict.

\section{Environmental Change}

Nothing is more important than environment as far as organizational change is concerned. This is because environment includes a wide range of factors as mentioned above. Even the areas of structures and cultures, which have been discussed separately in the previous sections, should be regarded as one of the environmental factors. As confirmed by Mullins (1999), changes in an organizational environment can be the source of major areas of conflict. By environment, he means organizations' external environment such as shifts in demand, increased competition, government intervention, new technology or 
changing social values. As has been proven in many cases, however, with their hard and restless efforts, some companies have been successful in turning the threatening situation into an opportunity to boost their performance through the competitive advantage resulting from the environmental turbulence.

The degree of conflict triggered by environmental change depends upon the levels of turbulence of the environment, as proposed by Ansoff and McDonnel (1990) as follows:

Level 1 : Predictable - a repetitive environment characterized by stability of markets; where the challenges repeat themselves; change is slower than the organisztion's ability to respond; the future is expected to be the same as the past.

Level 2 : Forecastable by extrapolation - complexity increases but managers can still extrapolate from the past and forecast the future with confidence.

Level 3 : Predictable threats and opportunities - complexity increases further when the organization's ability to respond becomes more problematic; however, the future can still be predicted with some degree of confidence.

Level 4 : Partially predictable opportunities - turbulence increases with the addition of global and socio-political changes. The future is only partly predictable.

Level 5 : Unpredictable Surprises - turbulence increases further with unexpected events and situations occurring more quickly than the organization can respond.

\section{Strategies for Managing Conflicts}

As the previous sections show, organizational conflict is obviously inevitable. When confronted to conflict, one can take any of the three optional approaches as suggested by Dinur (2011), namely: hit, run and stand. Whilst the hitter would deal with the conflict aggressively, the runner tends to avoid and to deny conflict, and the stander would try to acknowledge, understand and be willing to take active actions to handle the conflict Some strategies have been proposed for managing conflict that management can adopt to minimize 
its harmful effects. Mullins (1999) has proposed a list of ways of dealing with conflict as follows:

a) Clarification of goals and objective: misunderstandings can be avoided by the clarification and continual refinement of goals and objectives.

b) Resource distribution: managers may use their imagination and initiative to overcome conflict situations by sharing resources among their subordinates.

c) Personal policies and procedures: areas of conflict may be significantly reduced by careful and detailed attention to just and equitable personnel policies and procedures.

d) Non-monetary rewards: greater attention to non-monetary rewards such as, for example, more challenging works and greater empowerment, can be considered to reduce conflict when financial resources are limited.

e) Development of interpersonal/group process skills: to encourage a better understanding of one's own behavior, the other person's point of view, communication processes and problem solving, this kind of development may help encourage people to work through conflict situations in a constructive manner.

f) Group activities: dysfunctional conflict may be reduced by careful arrangement of the composition of groups and factors that affect group cohesiveness.

g) Leadership and management: conflict management will likely be eased by a more participative and supportive style of leadership.

h) Organizational processes: to reduce conflict situations, managers can pay special attention to such things as the nature of authority structure, work organization, patterns of communication and sharing information, and democratic functioning or organization.

i) Socio-technical approach: it is advised that organization should be viewed as a socio-technical system, which enables the development of psychological and social factors in appropriate to structural and technical requirements.

Mullins's formulations above are definitely prescribed for those who take the stand approach according to Dinur (2011). 


\section{Thomas's Model of Conflict-Handling Styles}

Thomas (1976) has developed a comprehensive model of conflict resolution behaviors as illustrated in Figure 1.

As is observed from the model, Thomas has identified five styles for handling conflict, namely:

1. Competitive or Domination. This style represents a desire to win one own concerns at the other's expense or to dominate.

2. Accommodative or appeasement. In contrast to competitive, this orientation focuses upon satisfying the other's concerns without attending to one's own.

Figure 1: Thomas's model of conflict-handling styles

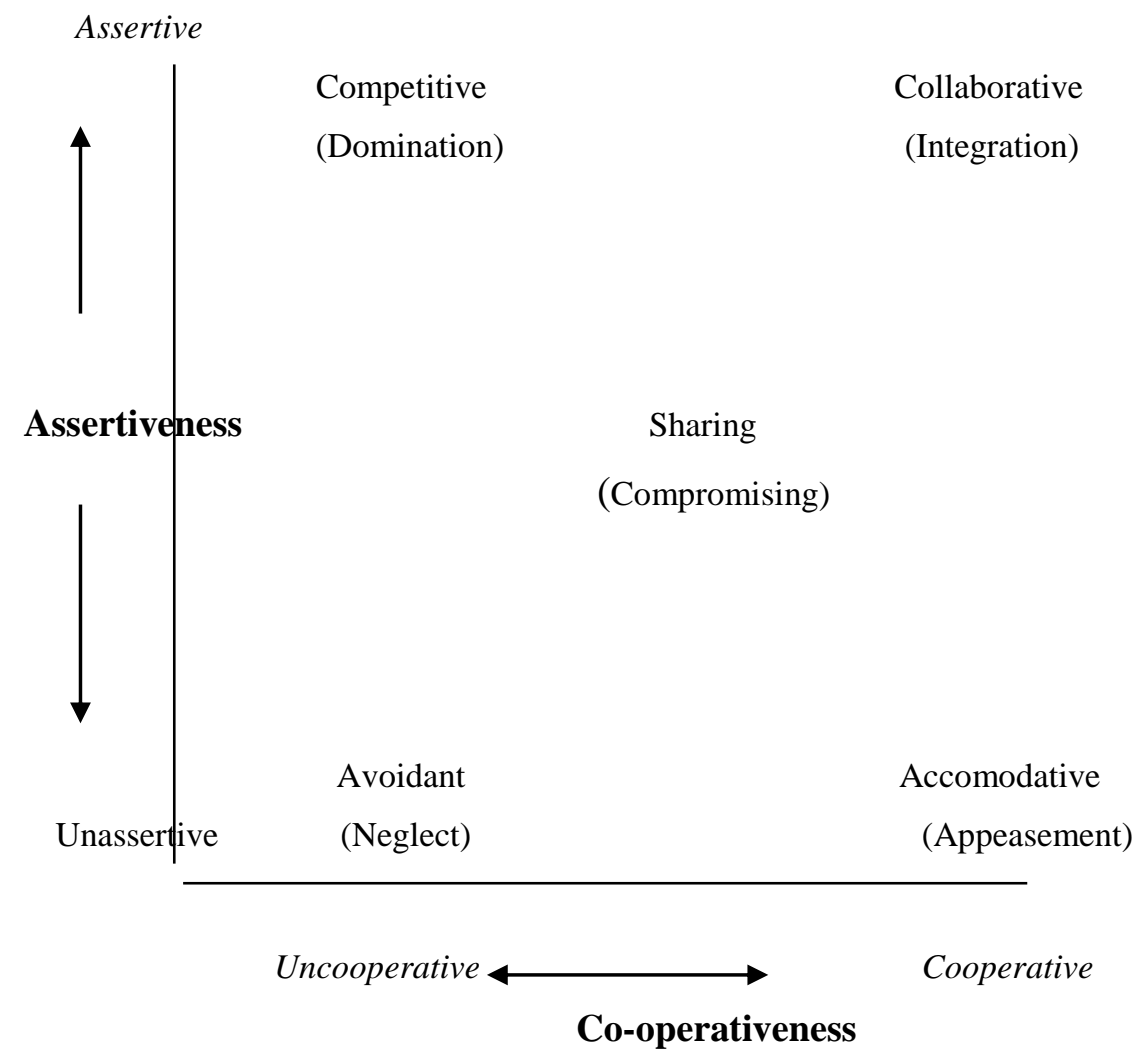


3. Sharing or Compromise. This is intermediate between domination and accommodative. It partially satisfies the needs of both parties, a preference for moderate.

4. Collaborative or Integration. In contrast to sharing, this style represents a desire to fully satisfy the concerns of both parties by integrating their concerns.

5. Avoidance or Neglect. This orientation reflects indifference to the concerns of either party. It creates a no-win situation because of ignorance or withdrawal from the conflict.

\section{Implications of Managing Conflict for Managers}

As an application of Thomas's model of conflict-handling styles, Robbins (1994) has come up with advice for managers who need to handle excessive conflict they are faced with as follows.

a) In emergencies, when quick, decisive action is needed use competitive style. This is applicable on important issues, where unpopular actions such as in cost cutting, and enforcing discipline need implementing.

b) When both sets of concerns are too important to be compromised, use collaborative style to find an integrative solution.

c) When an issue is trivial, or more important issues are pressing, that is when potential disruption overweighs the benefits of resolution, use avoidant style. When you find you are wrong and to allow a better position to be heard, when issues are more important to others than yourself and to satisfy others and maintain cooperation, use accommodative style.

d) Finally, when goals are important, but not worth the effort of potential disruption of more assertive approaches, use sharing or compromise style.

\section{Conclusions}

The discussions in this essay have led to the view that when organizational change is to be undertaken, conflict between individuals and groups is inevitable, and therefore should be accepted and handled properly in order to minimize its destructive effects. This is actually the current trend in 
viewing conflict in organizations, i.e., the view that conflict is not necessarily a bad thing and is unavoidable part of organizational process and development (the human relation approach), particularly in the times of change. However, such a view does ignore the thought of another approach, i.e., the traditional approach, which takes the view that conflict is totally destructive for organization, and therefore should be either avoided or completely treated. This view has definitely some value of truth in some way. Take as an example an organization which is in a complete turbulent situation, where the right and quick decision is vital, and no conflict at any level whatsoever is tolerable, or otherwise the organization collapses. In this kind of situation, the traditional approach must have the best resolution.

Regardless of such different views or approaches underlying the ideas, it is generally agreed that among other sources of conflict, environment is a very potential source of conflict. In its most turbulent and unpredictable condition, one can only rely on mostly his luck and a bit skills and experience to deal with the conflict resulting from it. However, even in its very turbulent form, with careful treatment and appropriate actions, environment may serve as a miracle that sometimes save organizations which under normal situation would likely collapse.

Finally, some formulations for conflict treatment have been proposed by various experts in the form of models or formulation. Among the prominent ones is the nine formulations proposed by Mullins (1999), i.e., clarification of goals and objectives, resource distribution, personnel policies and procedures, non-monetary rewards, development of interpersonal/group process skills, group activities, leadership and management, organizational processes, and socio-technical approach. A model of conflict resolution behaviors developed by Thomas (1976) may also be considered for an approach when coming to conflict problem solution. This model identifies five styles for handling conflict, namely: competitive or domination, accommodative or appeasement, sharing or compromise, collaborative or integration, and avoidance or neglect. Such kinds of prescription or formulation must obviously have some value for 
practitioner managers who are seeking a ready formulated treatment of a serious conflict they are confronted with, which crucially needs fast handling. However, it should be remembered that there is no one best medicine for all diseases, even if they are of the same kind and have the same symptoms. In the end, in a most critical situation, there could only one best way of treatment for conflict that emerges from that very specific situation.

\section{References}

Aldelfer, C.P., (1976), 'Change Processes in Organizations', in Dunnette M.D. (ed.), Handbook of Industrial and Organizational Psychology, Chicago: Rand McNally College Publishing Company.

Ansoff, H. Igor and McDonnell, Edward J., (1990), Implanting Strategic Management, London: Prentice Hall

Burnes, B., (1996), Managing Change, London: Pitman Publishing.

Child, J., (1976), Organization, London: Paul Chapman Publishing.

Dinur, Einav, (2011), Confronting Conflicts - A Toolbox for Understanding and Managing Conflicts, CISV International.

Drucker, P.F., (1974), "New templates for today's organizations," Harvard Business Review, January-February.

Hales, C., (1993), Managing through organisation, London: Routledge.

Handy, C., (1993), Understanding Organizations, London: Penguin.

Kanter, R.M., (1991), 'Transcending Business Boundaries: 12,000 World Managers View Change. Harvard Business Review, May-June.

Kanter, R.M., (1991), 'Championing Change: An Intrview With Bell Atlantic's CEO Raymond Smith', Harvard Business Review, January- February

Kotter, J.P., and Schleinger, L.A., (1991), 'Choosing Strategies for Change', Harvard Business Review, May-June.

Mullins, L.J., (1999), Management and Organisational Behaviour, London: Prentice Hall.

Robins, S.P., (1994), Essentials of Organizational Behavior, Englewood Cliffs, New Jersey: Prentice-Hall International.

Schelling, T.C., (1980), The Strategy of Conflict, Cambridge, Massachusettes: Harvard University.

Senior, B., (1997), Organisational Change, London: Prentice Hall.

Thomas, K., (1976), 'Conflict and Conflict Management', in Dunnette M.D. (ed.), Handbook of Industrial and Organizational Psychology, Chicago: Ran McNally College Publishing Company. 